ARTICLE

Received 13 Jul 2014 | Accepted 26 Jan 2015 | Published 3 Mar $2015 \quad$ DOl: 10.1038/ncomms7389

\title{
A quantum circuit rule for interference effects in single-molecule electrical junctions
}

David Zsolt Manrique ${ }^{1, \star}$, Cancan Huang ${ }^{2, \star}$, Masoud Baghernejad ${ }^{2, \star}$, Xiaotao Zhao $^{3, \star}$, Oday A. Al-Owaedi ${ }^{1,4, \star}$, Hatef Sadeghi ${ }^{1}$, Veerabhadrarao Kaliginedi ${ }^{2}$, Wenjing Hong ${ }^{2}$, Murat Gulcur ${ }^{3}$, Thomas Wandlowski ${ }^{2}$, Martin R. Bryce ${ }^{3} \&$ Colin J. Lambert ${ }^{1}$

A quantum circuit rule for combining quantum interference effects in the conductive properties of oligo(phenyleneethynylene) (OPE)-type molecules possessing three aromatic rings was investigated both experimentally and theoretically. Molecules were of the type $X-Y-X$, where $X$ represents pyridyl anchors with para $(p)$, meta $(m)$ or ortho (o) connectivities and $Y$ represents a phenyl ring with $p$ and $m$ connectivities. The conductances $G_{X m X}\left(G_{X p X}\right)$ of molecules of the form $X-m-X(X-p-X)$, with meta (para) connections in the central ring, were predominantly lower (higher), irrespective of the meta, para or ortho nature of the anchor groups $X$, demonstrating that conductance is dominated by the nature of quantum interference in the central ring $Y$. The single-molecule conductances were found to satisfy the quantum circuit rule $G_{\mathrm{ppp}} / G_{\mathrm{pmp}}=G_{\mathrm{mpm}} / G_{\mathrm{mmm}}$. This demonstrates that the contribution to the conductance from the central ring is independent of the para versus meta nature of the anchor groups.

\footnotetext{
${ }^{1}$ Department of Physics, Lancaster University, Lancaster LA1 4YB, UK. ${ }^{2}$ Department of Chemistry and Biochemistry, University of Bern, Freiestrasse 3 , Bern CH-3012, Switzerland. ${ }^{3}$ Department of Chemistry, Durham University, Durham DH1 3LE, UK. ${ }^{4}$ Department of Laser Physics, Woman College of Science, University of Babylon, Iraq. ${ }^{*}$ These authors contributed equally to this work. Correspondence and requests for materials should be addressed to W.H. (email: hong@dcb.unibe.ch) or to M.R.B. (email: m.r.bryce@durham.ac.uk) or to C.J.L. (email: c.lambert@lancaster.ac.uk)
} 
S tudies of the electrical conductance of single molecules attached to metallic electrodes not only probe the fundamentals of quantum transport but also provide the knowledge needed to develop future molecular-scale devices and functioning circuits $^{1-9}$. Owing to their small size (on the scale of Angstroms) and the large energy gaps (on the scale of eV), transport through single molecules can remain phase coherent even at room temperature, and constructive or destructive quantum interference (QI) can be utilized to manipulate their room temperature electrical ${ }^{10-13}$ and thermoelectrical ${ }^{14,15}$ properties. In previous studies, it was reported theoretically and experimentally that the conductance of a phenyl ring with meta (m) connectivity is lower than the isomer with para (p) connectivity by several orders of magnitude ${ }^{16-25}$. This arises because partial de Broglie waves traversing different paths through the ring are perfectly out of phase leading to destructive QI in the case of meta coupling, while for para or ortho coupling they are perfectly in phase and exhibit constructive QI. (See, for example, equation 8 of ref. 26.) It is therefore natural to investigate how QI in molecules with multiple aromatic rings can be utilized in the design of more complicated networks of interference-controlled molecular units.

The basic unit for studying QI in single molecules is the phenyl ring, with thiol ${ }^{17,21}$, methyl thioether ${ }^{27}$, amine ${ }^{17}$ or cyanide ${ }^{19}$ anchors directly connecting the aromatic ring to gold electrodes. Recently, Arroyo et al. ${ }^{28,29}$ studied the effect of QI in a central phenyl ring by varying the coupling to various anchor groups, including two variants of thienyl anchors. However, the relative importance of QI in central rings compared with QI in anchor groups has not been studied systematically because the thienyl anchors of Arroyo et al. ${ }^{28,29}$ were five-membered rings, which exhibit only constructive interference. To study the relative effect of QI in anchors, we examined molecules with terminal groups formed from six-membered pyridyl rings for which constructive and destructive interference is possible. We have previously reported that pyridyl rings are excellent anchor groups for attaching single molecules to metallic electrodes because of their well-defined binding geometry ${ }^{30}$, in which the nitrogen plays the role of the anchoring site. The ability to substitute pyridine either para (p), meta (m) or ortho (o) to the nitrogen offers the possibility of systematically investigating the relative importance of QI in this anchor group in molecules of the type X-Y-X, where $\mathrm{X}$ is a pyridyl ring and $\mathrm{Y}$ is a central phenyl ring. This question is rather subtle because charge transport from the electrode into the pyridyl rings takes place via the $\mathrm{N}$ atom and also via metal- $\pi$ coupling $^{31}$ and the interplay between these two mechanisms will determine the importance and robustness of QI in the terminal ${ }^{32}$ rings. In the present paper, our aim is to compare the effects of QI in both the terminal rings $\mathrm{X}$ and the central ring $\mathrm{Y}$ of molecules of

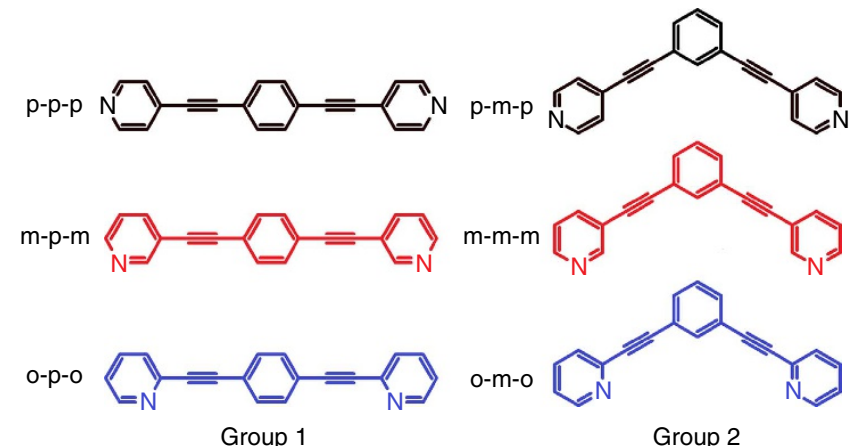

Figure 1 | The molecular structures studied in this paper. These are divided into two groups, based on the presence of a para (group 1) or meta (group 2) central phenyl ring. the type X-Y-X, and to study the relationship between their conductances. Our results were found to satisfy the quantum circuit rule $G_{\mathrm{ppp}} / G_{\mathrm{pmp}}=G_{\mathrm{mpm}} / G_{\mathrm{mmm}}$, which demonstrates that the contribution to the conductance from the central ring is independent of the para versus meta nature of the anchor groups. Combinatorial rules for QI in molecules with other geometries including fused rings or rings connected in parallel are discussed in refs 33-36.

We studied the pyridyl-terminated oligo(phenyleneethynylene) (OPE) derivatives shown in Fig. 1, which possess a variety of connectivities of the central ring and locations of the nitrogen in the anchor units. In group 1, the central unit $\mathrm{Y}$ is a phenyl ring with para connectivity, whereas the central ring of group 2 has meta connectivity. The anchor units $\mathrm{X}$ are pyridyl rings with their nitrogens located in either meta, ortho or para positions.

\section{Results}

Break-junction experiments. Charge transport characteristics of single-molecule junctions formed from the molecules in Fig. 1 were investigated using both the mechanically controllable breakjunction (MCBJ) and scanning tunnelling microscopy breakjunction (STM-BJ) techniques, as reported elsewhere $6,30,37,38$. Nuclear magnetic resonance spectra for these molecules and details of synthesis are presented in Supplementary Note 1 and Supplementary Figs 1-6. Figure 2a displays typical traces of the conductance $G$ (in units of the conductance quantum $G_{0}=2 e^{2} / h$ ) versus the relative electrode displacement $(\Delta z)$ from measurement of the molecule p-p-p. Corresponding results for other molecules are presented in Supplementary Note 2 and Supplementary Figs 7-12. $\Delta z$ is defined to be zero when $G=0.7$ $G_{0}$. It is related to the electrode separation $z_{\text {exp. }}$ by $z_{\text {exp. }}=\Delta z+\Delta z_{\text {corr }}$, where the correction $\Delta z_{\text {corr }}=0.5 \pm 0.1 \mathrm{~nm}$ accounts for the snap-back of the electrodes upon breaking of the gold-gold atomic contact ${ }^{39}$. For this molecule, the $\log \left(G / G_{0}\right)$ versus $\Delta z$ stretching traces possess well-defined plateaus in the range of $\log \left(G / G_{0}\right)$ around -4.5 , which we assign to the conductance of single-molecule junctions. The two-dimensional (2D) histograms of p-p-p in Fig. $2 b$ show features of gold point contacts around $G \geq 1 G_{0}$ followed by a second accumulation in the cloud-like scatter plot in the range $\left(10^{-5.0} G_{0}<G<10^{-3.6}\right.$ $G_{0}$ ), centred at $G=10^{-4.5} G_{0}$. We attribute the latter to the formation of single-molecule junctions. These clouds of conductance data lead to peaks in the corresponding onedimensional (1D) conductance histogram. The cloud-like pattern is observed in both MCBJ and STM-BJ measurements and the 1D histogram peaks are in good agreement with each other. Figure 2c,d displays the corresponding $1 \mathrm{D}$ conductance histograms of molecules belonging to groups 1 and 2 in a semilogarithmic scale, constructed from 1,000 experimental conductance-distance traces for each compound.

The sharp peaks $\sim 1 G_{0}$ represent the conductance of a singleatom gold-gold contact. The prominent peaks between $10^{-7}$ $G_{0}<G<10^{-4} G_{0}$ represent molecular conductance features. Significant difference of single-molecule conductances are observed from the variety of connectivities of the central ring (Fig. 2c) and locations of the nitrogen in the anchor units (Fig. 2d), while para connection in both central and terminal rings shows the highest conductance in both cases. The statistically most-probable conductance values were obtained by fitting Gaussians to the maxima in the conductance histograms. Our key results are summarized in Table 1. In anticipation of the theoretical discussion below, it is interesting to note that to within experimental error, $\log \left(G_{\mathrm{ppp}} / G_{0}\right)+\log \left(G_{\mathrm{mmm}} / G_{0}\right.$; that is, -4.5 to 6.9$)$ is equal to $\log \left(G_{\mathrm{pmp}} / G_{0}\right)+\log \left(G_{\mathrm{mpm}} / G_{0}\right.$; that is, -5.5 to 6.0 ), which suggests that the product of the 

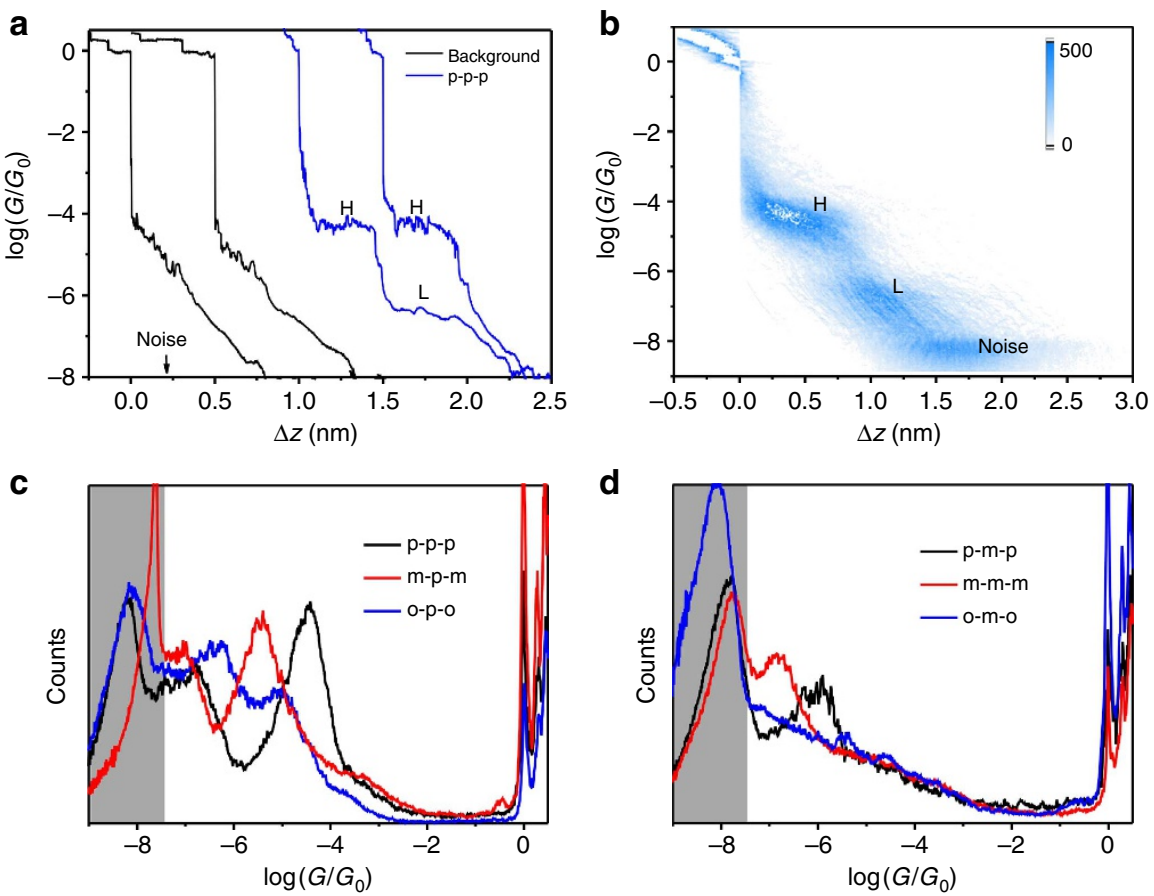

Figure 2 | Conductance measurements. (a) Typical individual conductance-distance traces of p-p-p (blue) and pure tunnelling traces (black). (b) All-data-point 2D conductance versus relative distance ( $\Delta z$ ) of p-p-p. (c,d) All-data-point 1D conductance histograms constructed from 1,000 MCBJ traces of molecules in group 1 (c) and in group 2 (d). The grey area is the noise level.

Table 1 | Most-probable experimental conductance, electrode separation $z_{\mathrm{H}}^{\star}$ at the end of the high-conductance plateaus and JFP of pyridyl-terminated OPE derivatives from MCBJ and STM-BJ.

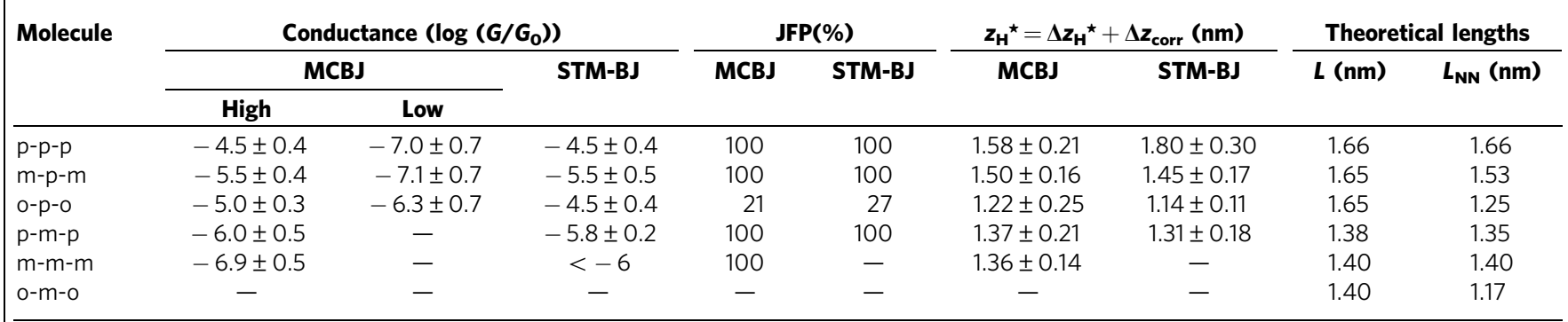

JFP, junction formation probability; MCBJ, mechanically controllable break-junction; OPE, oligo(phenyleneethynylene); STM-BJ, scanning tunnelling microscopy break-junction

The origin of differences between MCBJ and STM-BJ results are discussed in Supplementary Note 4. Error bars were determined from the standard derivation in Gaussian fitting of conductance and $\Delta z_{\mathrm{H}}{ }^{*}$ distribution. Comparison between theoretical lengths and most-probable end-of-plateau experimental electrode separations $z_{\mathrm{H}^{*}}$. The electrode separation $z_{\mathrm{H}}{ }^{*}$ is closer to the theoretical $\mathrm{N}---\mathrm{N}$ distance $L_{N N}$ than to the theoretical molecular length $L$.

conductances for $\mathrm{p}-\mathrm{p}-\mathrm{p}$ and $\mathrm{m}-\mathrm{m}-\mathrm{m}$ molecules is equal to the product of conductances for $\mathrm{p}-\mathrm{m}-\mathrm{p}$ and $\mathrm{m}-\mathrm{p}-\mathrm{m}$ molecules, and the quantum circuit rule $G_{\mathrm{ppp}} / G_{\mathrm{pmp}}=G_{\mathrm{mpm}} / G_{\mathrm{mmm}}$ is satisfied.

Further statistical analysis of conductance versus $\Delta z$ curves provides information about the junction formation probability (JFP) and allows us to determine the most-probable relative electrode displacement $\left(\Delta z_{\mathrm{H}}{ }^{*}\right)$ at the end of the high-conductance plateaus. For every $\log \left(G / G_{0}\right)$ versus $\Delta z$ stretching trace, we determine the relative electrode displacement at the end of the high-conductance plateau, $\Delta z_{\mathrm{H}}$, which is the largest $\Delta z$ value within the range $-0.3>\log \left(G / G_{0}\right)>\log \left(G_{\mathrm{H}}^{\text {end }} / G_{0}\right)$, where $G_{\mathrm{H}}^{\text {end }}$ is the end of high-conductance feature. The most-probable values of $\Delta z_{\mathrm{H}}$ (denoted $\left.\Delta z_{\mathrm{H}}{ }^{*}\right)$ are obtained by constructing a histogram and fitting a Gaussian function to the largest maxima. Taking into account the snap-back length, the most-probable electrode separations at the end of the high-conductance plateau are $z_{\mathrm{H}}{ }^{*}=$ $\Delta z_{\mathrm{H}}{ }^{*}+\Delta \mathrm{z}_{\text {corr }}$. The representative $\Delta z_{\mathrm{H}}$ histograms with Gaussian fitting functions are shown in Supplementary Figs $7-12$. The JFP is calculated as the ratio of the area under the fitted Gaussian function and the total area of the $\Delta z_{\mathrm{H}}$ histogram. If no distinct peak is observed in the $\Delta z_{\mathrm{H}}$ histogram, then the JFP is considered to be zero. Table 1 also summaries the various distances obtained from both MCBJ and STM-BJ measurements. As shown in Table 1, the JFP approaches $100 \%$ for molecules p-p-p, p-m-p, $\mathrm{m}-\mathrm{p}-\mathrm{m}$ and $\mathrm{m}-\mathrm{m}-\mathrm{m}$. For molecule $\mathrm{o}-\mathrm{p}-\mathrm{o}$, the JFP decreased sharply to $21 \%$ (MCBJ)/27\% (STM-BJ) because the $\mathrm{N}$ in the terminal ortho pyridyl is partially hidden from the electrode surfaces and therefore it is difficult to form a bridge between the two gold electrodes ${ }^{40}$. Our inability to measure the conductance of $\mathrm{o}-\mathrm{m}-\mathrm{O}$ is explained by its short $\mathrm{N}---\mathrm{N}$ length and the expected low conductance, which falls below the direct tunnelling conductance (Supplementary Note 3 and Supplementary Fig. 13).

The most-probable end-of-plateau electrode separations $z_{\mathrm{H}}{ }^{*}$ follow the trends $\mathrm{p}-\mathrm{p}-\mathrm{p}>\mathrm{m}-\mathrm{p}-\mathrm{m}>\mathrm{o}-\mathrm{p}-\mathrm{o}$, and $\mathrm{p}-\mathrm{m}-\mathrm{p} \approx \mathrm{m}-\mathrm{m}-\mathrm{m}$ that correlate with the molecular $\mathrm{N}---\mathrm{N}$ distance, demonstrating that the gold-anchor link is primarily controlled by the 


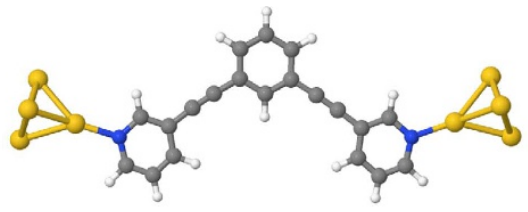

$\mathrm{mb}-\mathrm{m}-\mathrm{mb}$

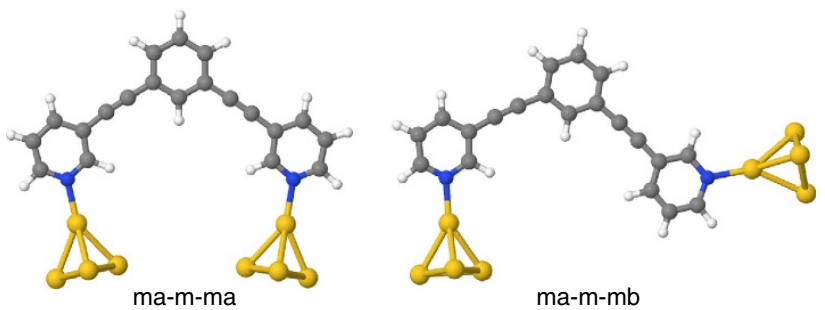

Figure 3 | Molecular conformations. Idealized junction geometries for the planar $\mathrm{m}-\mathrm{m}-\mathrm{m}$ molecule are shown. The $\mathrm{N}---\mathrm{N}$ distance varies with conformations, therefore, the molecular $\mathrm{N}---\mathrm{N}$ distance, $L_{\mathrm{NN}}$ is defined as the largest $\mathrm{N}---\mathrm{N}$ distance (mb-m-mb junction geometry).

gold-nitrogen bonds. Therefore, it is clear that changes in the position of the $\mathrm{N}$ atom within the anchors affects both the plateau length and the JFP, as well as the conductance.

Quantum interference in the terminal and central rings. For the linear molecules of group 1, both p-p-p and o-p-o have the same conductance value $\left(10^{-4.5} G_{0}\right)$ from the STM-BJ technique. The conductance value of o-p-o from MCBJ with the Gaussian fitting is $10^{-5.0} G_{0}$, which is lower than that obtained using the STM-BJ. We also note that the width of the conductance peak in the $1 \mathrm{D}$ histogram from MCBJ measurements is bigger than that from the STM-BJ. This is associated with the different stretching of the molecular junctions during their formation using the two techniques, as verified by the $2 \mathrm{D}$ conductance properties (see Supplementary Information). Nevertheless, compared with the STM-BJ results, the peak value from Gaussian fitting to MCBJ measurements is lower. These results suggest that a wider variety of configurations were formed between the molecule and electrodes during junction stretching in MCBJ measurements because of the higher stability, while some of the molecular configurations, especially the fully stretched configurations, could be monitored using the STM-BJ. The conductance of the m-p-m molecule was found to be lower than the other two group-1 members, with o-p-o and p-p-p in both the STM-BJ and MCBJ experiments, having a measured value of $10^{-5.5} G_{0}$.

Comparisons between the conductances of molecules in group 2 also demonstrate that the para/meta/ortho variations in the terminal ring result in only small conductance changes of less than an order of magnitude. The measured conductance of $\mathrm{m}-\mathrm{m}$ $\mathrm{m}$ is $10^{-6.9} G_{0}$, which is lower than the $\mathrm{p}-\mathrm{m}-\mathrm{p}$ conductance of $10^{-6.0} G_{0}$. However, as mentioned above, we were unable to measure any electrical properties of o-m-o from either MCBJ or STM-BJ owing to the masking direct tunnelling current. For comparison, in ref. 41 , changing from para to meta in the central ring of an OPV3 caused the conductance to drop by one order of magnitude, while in ref. 32 changing the position of an -SMe or an $-\mathrm{NH}_{2}$ in the anchor groups from para to meta caused a change of almost two orders of magnitude. These are different anchor groups from ours, but the conductance changes are comparable with our experimental observations.

To elucidate the measured conductance trends, we performed a density functional theory (DFT)-based optimization of each molecule using SIESTA ${ }^{42,43}$ and carried out electron transport

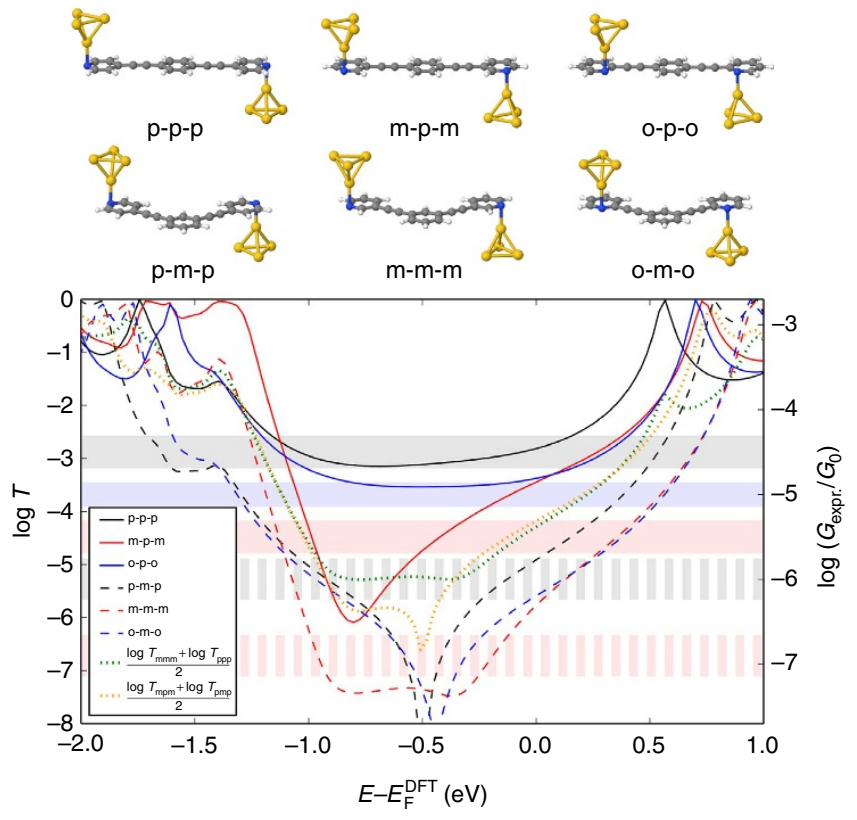

Figure 4 | Systematic junction geometries and transmission coefficient functions. The top figures show idealized junction geometries for all six molecules with the gold tip attached perpendicular to the pyridine ring. The bottom graphs show corresponding transmission coefficient curves. For comparison, the coloured and patterned horizontal shaded bars show the experimentally measured MCBJ values of $\log \left(G / G_{0}\right)$ (right-hand scale).

The thickness of the bars corresponds to the error bar of the experimental $\log \left(G / G_{0}\right)$. These show that both the transmission coefficients around the $E_{\mathrm{F}}$ and the experimental conductance values are separable into two groups corresponding to $\mathrm{X}-\mathrm{p}-\mathrm{X}$ and $\mathrm{X}-\mathrm{m}-\mathrm{X}$ connectivities. The green (yellow) dotted line is the average of $\log T_{\mathrm{mmm}}$ and $\log T_{\mathrm{ppp}}\left(\log T_{\mathrm{mpm}}\right.$ and $\left.\log T_{\mathrm{pmp}}\right)$. It should be noted that over most of the energy range from -0.8 to $-0.4 \mathrm{eV}$, the $\mathrm{m}-\mathrm{m}-\mathrm{m}$ results have the lowest transmission coefficient. At these low transmissions, the contribution from the $\sigma$ channel becomes comparable with that of the $\pi$ channel and therefore in this energy range, the circuit rule is violated.

calculations using the approach discussed in our previous papers $^{30,39}$. Unlike the $\mathrm{p}-\mathrm{p}$-p molecule, the other molecules possess anchoring nitrogen atoms located at meta or ortho positions within the terminal rings that do not naturally bind to planar electrode surfaces. To study the transport properties of these molecules, we attached the anchor nitrogen atoms to the apexes of pyramidal gold electrodes, as shown in Figs 3 and 4 and in Supplementary Note 5, and Supplementary Fig. 14. The molecules shown in these figures have been geometrically relaxed using SIESTA and it is clear that many of these geometries, such as the ma-m-ma conformation in Fig. 3, would not tend to form a bridge between two opposing pyramids. We also note that molecular lengths $(L)$, defined as the distance between the centres of the furthest non-hydrogen atoms in the molecule, are the same for these different conformations, whereas the $\mathrm{N}---\mathrm{N}$ distance, which is the distance between the centres of the $\mathrm{N}$ atoms, does vary. Therefore, in Table 1 the largest $\mathrm{N}---\mathrm{N}$ distance $\left(L_{\mathrm{NN}}\right)$ of each molecule is calculated and compared with the most-probable electrode separation at the end of the high-conductance plateau, that is, $z_{\mathrm{H}}{ }^{*}$.

The central focus of this section is to understand the relative contribution to the conductance from QI in the terminal and central aromatic rings, and to demonstrate that the quantum circuit rule is satisfied at the level of DFT. The largest value of $z_{\mathrm{H}}{ }^{*}$ among all the measured molecules (Table 1) was found to be 
$z_{\mathrm{H}}{ }^{*}=1.80 \mathrm{~nm}$ for the $\mathrm{p}-\mathrm{p}-\mathrm{p}$. The value of $z_{\mathrm{H}}{ }^{*}$ for other molecules was typically shorter than this value. Therefore, the typical conductances occur when the molecules are not fully stretched and there is a possibility of metal-ring overlap between the gold electrode and the pyridyl rings. To analyse such events, we systematically constructed junction geometries with two 35-atom gold (111)-directed pyramids attached to the $\mathrm{N}$ atoms perpendicular to the pyridyl rings, as shown in Fig. 4, and then computed the transmission coefficient curves $T(E)$ for these geometries. (Alternative junction geometries are investigated in Supplementary Note 6 and Supplementary Figs 15-17. Supplementary Figure 18 shows a comparison between theoretical and experimental conductances.)

Figure 4 demonstrates that for a wide range of Fermi energy choice, the theoretical and experimental conductances of the $\mathrm{X}$-p-X molecules of group 1 are distinctly higher than those of the molecules in group 2, as expected from previous studies ${ }^{27,28,32,41}$. Figure 4 also shows that for wide range of energies in the gap between the highest occupied molecular orbital (HOMO) and lowest unoccupied molecular orbital (LUMO), the ordering of the transmission coefficients follows the experimental conductance ordering. To demonstrate the resilience of the circuit rule, dotted lines are plots of $\left(\log T_{\mathrm{mmm}}+\log T_{\mathrm{ppp}}\right) / 2$ and $\left(\log T_{\mathrm{mpm}}+\log \right.$ $\left.T_{\mathrm{pmp}}\right) / 2$. The similarity of these two curves shows that the product rule is satisfied over a wide range of energies within the HOMO-LUMO gap.

To demonstrate that QI effects associated with variations in the positions of the $\mathrm{N}$ atoms are suppressed owing to the presence of a parallel conductance path associated with the electrode-ring overlap, we performed transport calculations in which we artificially set to zero every Hamiltonian and overlap matrix element that couples carbon and hydrogen atoms to gold atoms, leaving couplings between the nitrogen atoms and gold as the only possible transport path. The resulting transmission coefficients are shown as dashed lines in Fig. 5 and demonstrate that without metal-ring interactions the meta link in the terminal ring of the $\mathrm{m}-\mathrm{p}-\mathrm{m}$ molecule reduces the conductance by orders of magnitude, which is comparable with the effect of a meta link in the central ring, whereas in the presence of metal-ring interactions meta coupling in the terminal rings has a much smaller effect.

Derivation of the quantum circuit rule for molecular conductances. Having demonstrated that the effect of QI in a given ring depends on the position of the ring within the

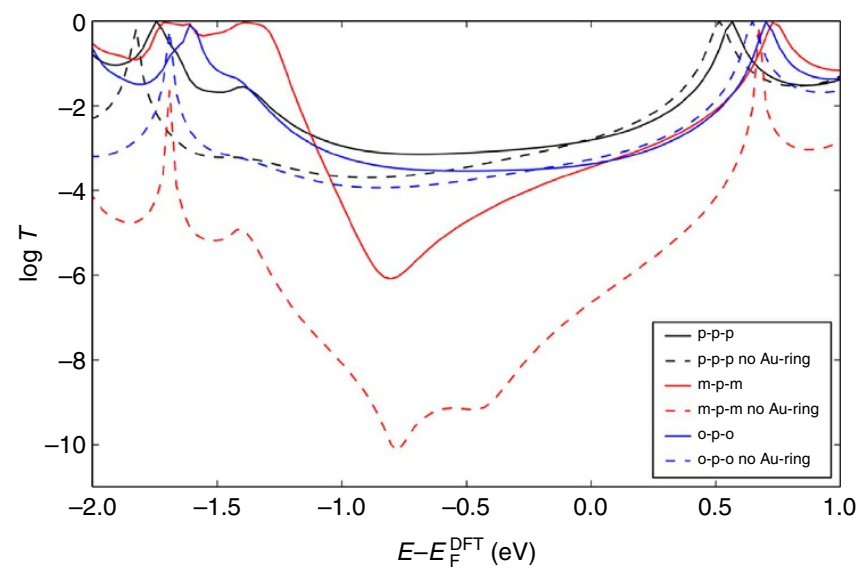

Figure 5 | Transmission curves with and without metal-ring coupling. The corresponding junction geometries are shown in Fig. 4. Dashed lines are without metal-ring coupling, the continuous curves are with metal-ring coupling. molecule (that is, whether it is a terminal or a central ring), we now derive the following quantum circuit rule describing the conductances of different ring combinations:

$$
G_{\mathrm{ppp}} \times G_{\mathrm{mmm}}=G_{\mathrm{pmp}} \times G_{\mathrm{mpm}}
$$

This captures the property that the contribution to the conductance from the ring $\mathrm{Y}$ in molecules of type $\mathrm{p}-\mathrm{Y}-\mathrm{p}$ and $\mathrm{m}-\mathrm{Y}-\mathrm{m}$ are identical.

To derive the circuit rule, consider the simple tight-binding model of Fig. 6b, in which three rings (labelled A, B and C) are coupled by nearest neighbour couplings $\alpha$ and $\beta$. To calculate the transmission coefficient arising when $1 \mathrm{D}$ electrodes are connected to site $\mathrm{i}$ of ring $\mathrm{A}$ and $\mathrm{q}$ of ring $\mathrm{C}$, we first calculate the Green's function $G_{\mathrm{qi}}$ of the whole structure in the absence of the electrodes.

This structure is an example of three arbitrary-coupled quantum objects shown schematically in Fig. 6a, whose Green's function is given by

$$
\begin{aligned}
& {\left[\begin{array}{ccc}
E-H_{\mathrm{A}} & -V_{\mathrm{AB}} & 0 \\
-V_{\mathrm{AB}}^{\dagger} & E-H_{\mathrm{B}} & -V_{\mathrm{BC}} \\
0 & -V_{\mathrm{BC}}^{\dagger} & E-H_{\mathrm{C}}
\end{array}\right]\left[\begin{array}{ccc}
G_{\mathrm{AA}} & G_{\mathrm{AB}} & G_{\mathrm{AC}} \\
G_{\mathrm{BA}} & G_{\mathrm{BB}} & G_{\mathrm{BC}} \\
G_{\mathrm{CA}} & G_{\mathrm{CB}} & G_{\mathrm{CC}}
\end{array}\right]} \\
& =I .
\end{aligned}
$$

Electron propagation from subsystem A to subsystem $\mathrm{C}$ is described by the block $G_{\mathrm{CA}}$, for which the above equation yields

$$
G_{\mathrm{CA}}=g_{\mathrm{C}} V_{\mathrm{C}}^{\dagger} G_{\mathrm{BB}} V_{\mathrm{A}}^{\dagger} g_{\mathrm{A}},
$$

where $g_{X}=\left(E-H_{X}\right)^{-1}(X=\mathrm{A}$ or $\mathrm{C})$ and $G_{\mathrm{BB}}=\left(E-H_{\mathrm{B}}-\right.$ $\left.V_{\mathrm{AB}}^{\dagger} g_{\mathrm{A}} V_{\mathrm{AB}}-V_{\mathrm{BC}} g_{\mathrm{C}} V_{\mathrm{BC}}^{\dagger}\right)^{-1}$. As an example, for the tight-binding model of Fig. 6b, this becomes $G_{q i}=\left(g_{\mathrm{C}}\right)_{\mathrm{qp}} B_{\mathrm{nm}}\left(g_{\mathrm{A}}\right)_{\mathrm{ji}}$, where $B_{\mathrm{nm}}=\beta \frac{\left(g_{B}\right)_{\mathrm{nm}}}{(1-\sigma)}$ and $\sigma=\alpha^{2}\left(g_{\mathrm{A}}\right)_{\mathrm{jj}}\left(g_{B}\right)_{\mathrm{mm}}+\beta^{2}\left(g_{\mathrm{C}}\right)_{\mathrm{pp}}\left(g_{\mathrm{B}}\right)_{\mathrm{nn}}+\alpha^{2} \beta^{2}$ $\left(g_{\mathrm{A}}\right)_{\mathrm{jj}}\left(g_{\mathrm{C}}\right)_{\mathrm{pp}}\left[\left(g_{\mathrm{B}}\right)_{\mathrm{nm}}\left(g_{\mathrm{B}}\right)_{\mathrm{mn}}-\left(g_{\mathrm{B}}\right)_{\mathrm{nn}}\left(g_{\mathrm{B}}\right)_{\mathrm{mm}}\right]$.

The crucial point is that $B_{\mathrm{nm}}$ does not depend on the choice of $\mathrm{p}, \mathrm{q}, \mathrm{i}$ and $\mathrm{j}$, because due to rotational symmetry, $\left(g_{\mathrm{A}}\right)_{\mathrm{jj}}$ and $\left(g_{\mathrm{C}}\right)_{\mathrm{pp}}$ are independent of $j$ and $p$ for any choice of $j$ and $p$. This means that $B_{\mathrm{nm}}$ does not depend on the choice of meta, ortho or para coupling for rings $\mathrm{A}$ and $\mathrm{C}$ and only depends on the connectivity of ring $\mathrm{B}$. When the rings $\mathrm{A}$ and $\mathrm{C}$ are coupled to the electrodes, this rotational symmetry is broken slightly. However, provided $E_{\mathrm{F}}$ lies in the HOMO-LUMO gap of $\mathrm{A}$ and $\mathrm{C}$ and the coupling to the electrodes is small (as is usually the case for molecules attached to gold electrodes, where the level broadening is much less than the level spacing), then the symmetry breaking is weak ${ }^{26}$. Since the electrical conductance is proportional to $\left|G_{\mathrm{qi}}\right|^{2}$, this means that the electrical conductance $G_{X Y Z} / G_{0}$ of molecules of the type $\mathrm{X}-\mathrm{Y}-\mathrm{Z}$ is proportional to a product of the form $a_{\mathrm{X}} \times b_{\mathrm{Y}} \times a_{\mathrm{Z}}$ and hence the quantum circuit rule is satisfied. The factors $a_{\mathrm{X}}, b_{\mathrm{Y}}$,

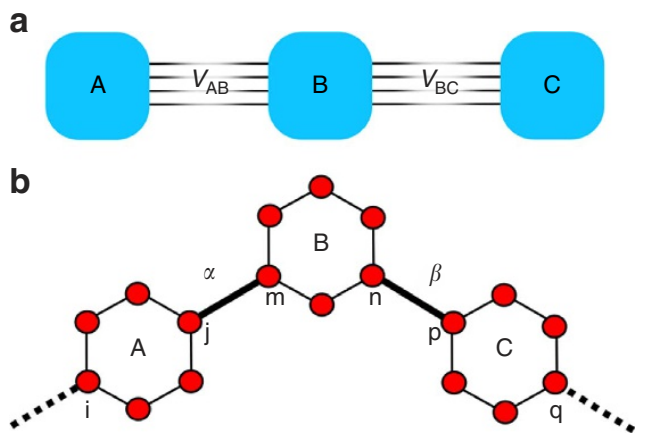

Figure 6 | Abstract models. (a) An abstract depiction of three coupled quantum subsystems. (b) A simple tight-binding model of the three ring system. (In the figure $p-m-p$ connection is shown to illustrate the connecting sites $i, j, m, n, p$ and $q$.) 
$a_{\mathrm{Z}}$ are contributions to the overall conductance from the separate rings, but it should be noted that they are not themselves individually measureable conductances. The above analysis can be easily applied to a molecule with two rings yield the circuit rule $G_{\mathrm{pp}} G_{\mathrm{mm}}=G_{\mathrm{pm}}^{2}$, where the $G_{\mathrm{pp}}$ and $G_{\mathrm{mm}}$ are the conductances of the molecule with para-para and meta-meta connected rings, and $G_{\mathrm{pm}}$ is the conductance of the molecule with meta and para rings (see Supplementary Information for a DFT confirmation of this rule).

To check the experimental validity of the circuit rule (1) for the OPEs of Fig. 1, we examine the measured conductance values presented in Table 1. For example, rearranging equation (1) into the form $G_{\mathrm{pmp}}=\frac{G_{\mathrm{ppp}}}{G_{\mathrm{mpm}}} G_{\mathrm{mmm}}$ and substituting into the right-hand side, the measured values for $G_{\mathrm{ppp}}, G_{\mathrm{mmm}}$ and $G_{\mathrm{mpm}}$ yields $G_{\mathrm{pmp}}=10^{-5.9} G_{0}$, which compares well with the measured value of $G_{\mathrm{pmp}}=10^{-5.8} G_{0}$ shown in Table 1 .

\section{Discussion}

We have presented charge transport studies of pyridyl terminated OPE derivatives, using the MCBJ and STM-BJ techniques, DFT-based theory and analytic Green's functions, and have investigated the interplay between QI effects associated with central and terminal rings in molecules of the type X-Y-X.

Our results demonstrated that the contribution to the conductance from the central ring is independent of the para or meta nature of the anchor groups and the combined conductances satisfy the quantum circuit rule $G_{\mathrm{ppp}} / G_{\mathrm{pmp}}=$ $G_{\mathrm{mpm}} / G_{\mathrm{mmm}}$. For the simpler case of a two-ring molecule, the circuit rule $G_{\mathrm{pp}} G_{\mathrm{mm}}=G_{\mathrm{pm}}^{2}$ is satisfied (Supplementary Note 8, and Supplementary Figs 19 and 20). It should be noted that the circuit rule does not imply that the conductance $G_{X Y X}$ is a product of three measureable conductances associated with rings $\mathrm{X}, \mathrm{Y}$ and $\mathrm{X}$. Indeed the latter property does not hold for a single molecule. On the other hand, provided sample to sample fluctuations lead to a broad distribution of phases within an ensemble of measurements, a product rule for ensemble averages of conductances can arise. This possibility is discussed in detail in Supplementary Notes 7 and 9.

The qualitative relationship between the conductances agrees well with the simple QI picture of molecular conduction. It has been reported that destructive QI exists in benzene with the meta connectivity and is responsible for the observed reduction of conductance ${ }^{16,20,28}$, whereas for para and ortho connectivities, constructive QI should be observed ${ }^{44,45}$. The transmission coefficient calculations through junctions where the metal-ring connection is artificially blocked (Fig. 5) show that the artificially coupled pyridyl ring exhibits similar behaviour to the benzene ring, with destructive QI in the case of the meta coupling significantly reducing the conductance compared with para and ortho connectivities. The dashed curves in the bottom panel in Fig. 5 clearly demonstrate that when the conduction is through only the nitrogen atoms, the conductance of the meta isomer is much lower than in the para and ortho isomers. More realistically, however, in the presence of metal-ring overlap, the effect of varying the positions of the nitrogens in the anchors becomes much weaker, and as demonstrated by Fig. 4 the major changes in the molecular conductance are caused by the variations in the connectivity of the central ring. The dominant influence of the central ring is accounted for by the fact that the central ring is not in direct contact with electrodes and therefore no parallel conductance paths are present, which could bypass the ethynylene connections to the anchors.

In a subnanometre scale molecular circuit, as in standard complementary metal-oxide-semiconductor (CMOS) circuitry, electrical insulation is of crucial importance. Destructive interference in a two-terminal device may not be desirable, because of the lower conductance. However, for a three-terminal device minimizing the conductance of the third terminal is highly desirable, because the third (gate) electrode should be placed as close to the molecule as possible, but at the same time, there should be no leakage current between the molecule and gate. One way of achieving this may be to use an anchor group with built-in destructive interference. Therefore, destructive QI may be a vital ingredient in the design of future three-terminal molecular devices and more complicated networks of interference-controlled molecular units.

\section{Methods}

Conductance measurements. The transport characteristics in single-molecule junctions were studied by MCBJ and STM-BJ measurements in solution at room temperature. The latter contained typically $0.1 \mathrm{mM}$ of the OPE-type molecules in a mixture of 1,3,5-trimethylbenzene (Aldrich, p.a.) and tetrahydrofuran (Aldrich, p.a), $4: 1(\mathrm{v} / \mathrm{v})$.

MCBJ measurements. The MCBJ experiments are based on the formation and breaking of a nanogap between a notched, freely suspended gold wire $(0.1 \mathrm{~mm}$ diameter, $99.999 \%$, Goodfellow), fixed on spring steel sheets $(10 \mathrm{~mm} \times 30 \mathrm{~mm}$, thickness $0.25 \mathrm{~mm}$ ) with a two-component epoxy glue (Stycast $2850 \mathrm{FT}$ with catalyst 9). The sample sheets were fixed between two holders. A Kel-F liquid cell with a Kalrez O-ring was mounted onto the sheet. During the measurements, the steel sheet could be bent with a pushing rod, which was controlled by a combination of a stepper motor and a piezo stack. The bending was initialized by the stepper motor. Once the measured current decreased to a value corresponding to $15 G_{0}$, the stepper motor stops moving and the piezo stack was activated. This strategy reduced significantly noise contributions from the operation of the stepper motor. The movement of the piezo stack controlled the breaking and the reformation of nanoscale contacts, typically in the range between the noise threshold $\left(G<10^{-8} G_{0}\right)$ and a high-conductance limit, which was set to $10 G_{0}$. Molecular junctions could form upon breaking the gold-gold nanocontacts. The entire cycle was repeated for $>1,000$ times to obtain statistically relevant data. In the MCBJ setup, the current could be recorded as a feedback signal at a given bias voltage (typically between 0.020 and $0.200 \mathrm{~V}$ ). The two ends of the 'broken wire' were taken as working electrodes WE1 and WE2.

The MCBJ unit is controlled by a lab-built bipotentiostat with two bipolar tunable logarithmic $I-V$ converters as current measuring units, which are operated by a custom-designed microcontroller. The system provides the following three analogue signals: the potential of WE1, the voltage difference between the two working electrodes WE1 and WE2 (bias voltage $V_{\text {bias }}$ ), driving the current through the two gold electrodes for the conductance measurements, and the voltage output of the piezo stack in the range of $0-50 \mathrm{~V}$, allowing the displacement of the piezo stack up to $8 \mu \mathrm{m}$ with rates ranging from 3 to $3,000 \mathrm{~nm} \mathrm{~s}^{-1}$. The latter translates into lateral pulling (pushing) rates between the two gold leads of $0.1-100 \mathrm{~nm} \mathrm{~s}^{-1}$ The distance between the two gold electrodes in the MCBJ setup was calibrated with complementary STM-BJ experiments, assuming that the tunnelling decay is identical under the same experimental conditions. Further technical details of the MCBJ setup was reported by Hong et al. ${ }^{46}$

STM-BJ. The STM-BJ technique repeatedly traps molecules between a sharp STM tip and an atomically flat sample. The STM-BJ measurements were carried out with a molecular imaging PicoSPM housed in an all-glass argon-filled chamber and equipped with a dual preamplifier capable of recording currents in a wide range of $1 \mathrm{pA}$ to $150 \mu \mathrm{A}$ with high resolution. The non-amplified low-current signal was fed back to the STM controller preserving the STM imaging capability. The currentdistance measurements were performed with a separate, lab-built analogue ramp unit. For further technical details, we refer to our previous work 47,48

The sample electrode was gold single-crystal bead. The Au (111) facet before each experiment was subjected to electrochemical polishing and annealing in a hydrogen flame followed by cooling under Ar. A freshly prepared solution containing typically $0.1 \mathrm{mM}$ of the respective molecule was added to a Kel-F flowthrough liquid cell mounted on top of the sample, and the uncoated STM tip was electrochemically etched using gold wire (Goodfellow, 99.999\%, $0.25 \mathrm{~mm}$ diameter) capable of imaging with atomic resolution. This system relies on trapping a molecule between the end of an Au tip and the gold substrate.

After assembling the experiment, the following protocols were applied: the tip was brought to a preset tunnelling position typically defined by $i_{\mathrm{T}}=50-100 \mathrm{pA}$ and a bias voltage $V_{\text {bias }}$ of $0.10,0.065$ and $0.175 \mathrm{~V}$, followed by imaging the substrate.

After fixing the lateral position of the tip, the STM feedback is switched off and current-distance measurements were performed, and then the vertical movement of the tip was controlled by the ramp unit. The measuring cycle was performed in the following way: the controlling software drives the tip towards the adsorbatemodified surface. The approach was stopped when a predefined upper current limit was reached (typically $10 \mu \mathrm{A}$ or $<10 G_{0}$ with $G_{0}$ being the fundamental conductance quantum $77.5 \mu \mathrm{S})$. After a short delay $(\sim 100 \mathrm{~ms})$ ensuring tip 
relaxation and the formation of stable contacts, the tip was retracted by $2-5 \mathrm{~nm}$ until a low current limit of $\sim 10 \mathrm{pA}$ was reached. The approaching and withdrawing rates were varied from 56 to $145 \mathrm{~nm} \mathrm{~s}^{-1}$. The entire current-distance traces were recorded with a digital oscilloscope (Yokogawa DL 750, 16 bit, $1 \mathrm{MHz}$ sampling frequency) in blocks of 186 individual traces. Up to 2,000 traces were recorded for each set of experimental conditions to guarantee the statistical significance of the results. For each molecule, the data were acquired at three different bias voltages of $0.065,0.100$ and $0.175 \mathrm{~V}$.

Theory and simulations. All molecules in this study were first geometrically optimized in isolation. For molecules with meta and ortho terminal pyridyl rings, all the non-equivalent planar conformations were geometrically relaxed. All geometrical optimizations were carried out using the DFT code SIESTA, with a generalized gradient approximation method (PBE functional), double-zeta polarized basis set, $0.01 \mathrm{eV}^{-1}$ force tolerance and $250 \mathrm{Ry}$ mesh cutoff. To investigate ideal junction geometries, a small four-atom gold pyramid was attached to the $\mathrm{N}$ atoms of the molecules, with $\mathrm{Au}-\mathrm{N}-\mathrm{C}$ angle being $120^{\circ}$ and $\mathrm{Au}-\mathrm{N}$ bond length being 2.1 $\AA$, as shown in Supplementary Fig. 14. Many of these junction geometries are unlikely to happen in $\mathrm{BJ}$ experiments, and during the junction elongation typically the gap between the electrodes is shorter than the molecular length. We constructed another set of idealized junction geometries where the gold pyramid is attached to rings from the side, perpendicular to the ring. These junction geometries are shown in Fig. 4. For transport calculation, the four-atom gold pyramids that are seen in Fig. 4 are extended to a 35-atom gold pyramid. Transmission coefficients were computed using the GOLLUM code ${ }^{49}$.

In break-junction experiments, more complicated structures are expected. For this reason, we also carried out BJ simulations using the approach discussed in our previous papers ${ }^{30,39}$. The geometrically optimized molecules were inserted between two opposing 35-atom (111) directed pyramids with four different tip separations. (The tip separation is defined to be the centre to centre distance between the apex atoms of the two opposing pyramids.) In the initial geometries, the molecules were shifted slightly towards one of the pyramids and the initial $\mathrm{Au}-\mathrm{N}$ distances were $\sim 2.5 \AA$. Then the constructed structure was geometrically relaxed such that the base layers of the pyramids were kept fixed during the optimizations. Optimized junction geometries are shown in Supplementary Figs 16 and 17.

To produce the conductance-trace curves in Supplementary Fig. 18, the transmission coefficient $T(E)$ was calculated for each relaxed junction geometry, by first obtaining the corresponding Hamiltonian and then overlapping matrices with SIESTA and double-zeta polarized basis set. The transmission coefficient for all junction geometries in this study was calculated using wide-band electrodes with $\Gamma=4.0 \mathrm{eV}$. The wide-band electrodes were coupled to the two base layers of gold atoms of the 35-atom pyramids. A few additional transmission coefficient functions can be seen in Supplementary Fig. 15. To produce conductance-trace curves, the transmission coefficient $T(E)$ was calculated for each relaxed junction geometry and the conductance $G / G_{0}=T\left(E_{\mathrm{F}}\right)$ was obtained by evaluating $T(E)$ at the Fermi energy $E_{\mathrm{F}}$. A value of $E_{\mathrm{F}}=-0.65 \mathrm{eV}$, relative to the bare DFT Fermi energy $\left(E_{\mathrm{F}}^{\mathrm{DFT}}\right)$ was chosen to account for the expected LUMO level shift, as discussed in ref. 39, where the Fermi energy shift was adjusted to match measured and computed conductance for the pyridine-terminated oligoyne molecules.

Synthesis. Details of the synthesis are in the Supplementary Note 1.

\section{References}

1. Chen, J., Reed, M. A., Rawlett, A. M. \& Tour, J. M. Large on-off ratios and negative differential resistance in a molecular electronic device. Science 286, 1550-1552 (1999).

2. Reed, M. A., Zhou, C., Muller, C. J., Burgin, T. P. \& Tour, J. M. Conductance of a molecular junction. Science 278, 252-254 (1997).

3. Smit, R. H. M. et al. Measurement of the conductance of a hydrogen molecule. Nature 419, 906-909 (2002)

4. Xu, B. Q. \& Tao, N. J. Measurement of single-molecule resistance by repeated formation of molecular junctions. Science 301, 1221-1223 (2003).

5. Zhou, X.-S. \& Maisonhaute, E. in Electrochemistry: Volume 11-Nanosystems Electrochemistry, Vol. 11 (eds Wadhawan, J. D. \& Compton, R. G.) 1-33 (The Royal Society of Chemistry, 2013).

6. Jia, C. \& Guo, X. Molecule-electrode interfaces in molecular electronic devices. Chem. Soc. Rev. 42, 5642-5660 (2013)

7. Venkataraman, L., Klare, J. E., Nuckolls, C., Hybertsen, M. S. \& Steigerwald, M. L. Dependence of single-molecule junction conductance on molecular conformation. Nature 442, 904-907 (2006).

8. Diez-Perez, I. et al. Controlling single-molecule conductance through lateral coupling of pi orbitals. Nat. Nanotechnol. 6, 226-231 (2011).

9. Xiang, D. et al. Three-terminal single-molecule junctions formed by mechanically controllable break junctions with side gating. Nano Lett. 13, 2809-2813 (2013).

10. Chaste, J. et al. A nanomechanical mass sensor with yoctogram resolution. Nat. Nanotechnol. 7, 301-304 (2012).

11. Ballmann, S. et al. Experimental evidence for quantum interference and vibrationally induced decoherence in single-molecule junctions. Phys. Rev. Lett. 109, 056801 (2012).
12. Kaliginedi, V. et al. Correlations between molecular structure and singlejunction conductance: a case study with oligo (phenylene-ethynylene)-type wires. J. Am. Chem. Soc. 134, 5262-5275 (2012).

13. Guédon, C. M. et al. Observation of quantum interference in molecular charge transport. Nat. Nanotechnol. 7, 305-309 (2012).

14. Finch, C. M., Garcia-Suarez, V. M. \& Lambert, C. J. Giant thermopower and figure of merit in single-molecule devices. Phys. Rev. B 79, 033405 (2009).

15. Evangeli, C. et al. Engineering the thermopower of C60 molecular junctions. Nano Lett. 13, 2141-2145 (2013)

16. Ke, S.-H., Yang, W. \& Baranger, H. U. Quantum-interference-controlled molecular electronics. Nano Lett. 8, 3257-3261 (2008).

17. Kiguchi, M. \& Kaneko, S. Single molecule bridging between metal electrodes Phys. Chem. Chem. Phys. 15, 2253-2267 (2013).

18. Kiguchi, M., Nakamura, H., Takahashi, Y., Takahashi, T. \& Ohto, T. Effect of anchoring group position on formation and conductance of a single disubstituted benzene molecule bridging Au electrodes: change of conductive molecular orbital and electron pathway. J. Phys. Chem. C 114, 22254-22261 (2010).

19. Koga, J., Tsuji, Y. \& Yoshizawa, K. Orbital control of single-molecule conductance perturbed by $\pi$-accepting anchor groups: cyanide and isocyanide. J. Phys. Chem. C 116, 20607-20616 (2012).

20. Markussen, T., Stadler, R. \& Thygesen, K. S. The relation between structure and quantum interference in single molecule junctions. Nano Lett. 10, 4260-4265 (2010).

21. Mayor, M. et al. Electric current through a molecular rod-relevance of the position of the anchor groups. Angew. Chem. Int. Ed. 42, 5834-5838 (2003).

22. Okuno, Y. \& Ozaki, T. First-principles study of multiterminal quantum interference controlled molecular devices. J. Phys. Chem. C 117, 100-109 (2012).

23. Saha, K. K., Nikolić, B. K., Meunier, V., Lu, W. \& Bernholc, J. Quantum-interference-controlled three-terminal molecular transistors based on a single ring-shaped molecule connected to graphene nanoribbon electrodes. Phys. Rev. Lett. 105, 236803 (2010).

24. Cardamone, D. M., Stafford, C. A. \& Mazumdar, S. Controlling quantum transport through a single molecule. Nano Lett. 6, 2422-2426 (2006).

25. Rabache, V. et al. Direct observation of large quantum interference effect in anthraquinone solid-state junctions. J. Am. Chem. Soc. 135, 10218-10221 (2013).

26. Lambert, C. J. Basic concepts of quantum interference and electron transport in single-molecule electronics. Chem. Soc. Rev. 44, 875-888 (2015).

27. Aradhya, S. V. et al. Dissecting contact mechanics from quantum interference in single-molecule junctions of stilbene derivatives. Nano Lett. 12, 1643-1647 (2012).

28. Arroyo, C. R. et al. Signatures of quantum interference effects on charge transport through a single benzene ring. Angew. Chem. Int. Ed. 52, 3152-3155 (2013).

29. Arroyo, C. et al. Quantum interference effects at room temperature in OPV-based single-molecule junctions. Nanoscale Res. Lett. 8, 234 (2013).

30. Hong, W. et al. Single molecular conductance of tolanes: experimental and theoretical study on the junction evolution dependent on the anchoring group. J. Am. Chem. Soc. 134, 2292-2304 (2012).

31. Quek, S. Y. et al. Mechanically controlled binary conductance switching of a single-molecule junction. Nat. Nanotechnol. 4, 230-234 (2009).

32. Meisner, J. S. et al. Importance of direct metal $-\pi$ coupling in electronic transport through conjugated single-molecule junctions. J. Am. Chem. Soc. 134, 20440-20445 (2012).

33. Xia, J. et al. Breakdown of interference rules in azulene, a non-alternant hydrocarbon. Nano Lett. 14, 2941-2945 (2014).

34. Valkenier, H. et al. Cross-conjugation and quantum interference: a general correlation? Phys. Chem. Chem. Phys. 16, 653-662 (2013).

35. Vazquez, H. et al. Probing the conductance superposition law in singlemolecule circuits with parallel paths. Nat. Nanotechnol. 7, 663-667 (2012).

36. Taniguchi, M. et al. Dependence of single-molecule conductance on molecule junction symmetry. J. Am. Chem. Soc. 133, 11426-11429 (2011).

37. Hong, W. et al. An MCBJ case study: The influence of pi-conjugation on the single-molecule conductance at a solid/liquid interface. Beilstein J. Nanotechnol. 2, 699-713 (2011)

38. Kaliginedi, V. et al. Correlations between molecular structure and singlejunction conductance: a case study with oligo(phenylene-ethynylene)-type wires. J. Am. Chem. Soc. 134, 5262-5275 (2012).

39. Moreno-García, P. et al. Single-molecule conductance of functionalized oligoynes: length dependence and junction evolution. J. Am. Chem. Soc. 135, 12228-12240 (2013).

40. Wu, S. et al. Molecular junctions based on aromatic coupling. Nat. Nanotechnol. 3, 569-574 (2008).

41. Arroyo, C. R. et al. Quantum interference effects at room temperature in OPV-based single-molecule junctions. Nanoscale Res. Lett. 8, 1-6 (2013). 
42. Soler, J. M. et al. The SIESTA method for ab initio order-N materials simulation. J. Phys. Condens. Matter 14, 2745 (2002).

43. Artacho, E. et al. The SIESTA method; developments and applicability. J. Phys. Condens. Matter 20, 064208 (2008).

44. Hansen, T., Solomon, G. C., Andrews, D. Q. \& Ratner, M. A. Interfering pathways in benzene: an analytical treatment. J. Chem. Phys. 131, 194704 (2009).

45. Solomon, G. C. et al. Understanding quantum interference in coherent molecular conduction. J. Chem. Phys. 129, 054701 (2008).

46. Hong, W. et al. An MCBJ case study: The influence of $\pi$-conjugation on the single-molecule conductance at a solid/liquid interface. Beilstein J. Nanotechnol. 2, 699-713 (2011).

47. Mishchenko, A. et al. Influence of conformation on conductance of biphenyl-dithiol single-molecule contacts. Nano Lett. 10, 156-163 (2010).

48. Mishchenko, A. et al. Single-molecule junctions based on nitrile-terminated biphenyls: a promising new anchoring group. J. Am. Chem. Soc. 133, 184-187 (2011).

49. Ferrer, J. et al. GOLLUM: a next-generation simulation tool for electron, thermal and spin transport. New J. Phys. 16, 093029 (2014).

\section{Acknowledgements}

This work was generously supported by the Swiss National Science Foundation (200020-144471; NFP 62), the German Science Foundation (priority programme SPP 1243), the UK Engineering and Physical Research Council grants EP/K001507/1, EP/J014753/1 and EP/H035818/1, Ministry of Higher Education and Scientific Research of Iraq, the EC FP7 ITNs 'MOLESCO' and 'FUNMOLS' project numbers 606728 and 212942, respectively, and the University of Bern.

\section{Author contributions}

All authors provided essential contributions to the manuscript and the project. All authors have given approval to the final version of the manuscript. C.J.L., D.Z.M., T.W., W.H. and M.R.B. originally conceived the concept and designed the experiments. D.Z.M., C.H., W.H., M.R.B. and C.J.L. prepared the manuscript using feedback from other authors. Synthetic work was carried out in the laboratory of M.R.B. by X.Z. and M.G.; break-junction measurements were carried out in the laboratory of T.W. by C.H., M.B. and V.K.; and calculations were carried out in the laboratory of C.J.L. by D.Z.M., O.A.A.-O. and H.S.

\section{Additional information}

Supplementary Information accompanies this paper at http://www.nature.com/ naturecommunications

Competing financial interests: The authors declare no competing financial interests.

Reprints and permission information is available online at http://npg.nature.com/ reprintsandpermissions/

How to cite this article: Manrique, D. Z. et al. A quantum circuit rule for interference effects in single-molecule electrical junctions. Nat. Commun. 6:6389 doi: $10.1038 /$ ncomms7389 (2015). 